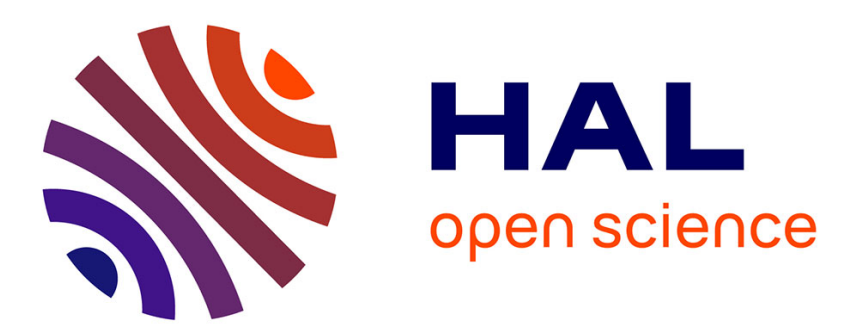

\title{
Effects of transverse heterogeneities on the most likely path of protons
}

Feriel Khellaf, Nils Krah, Ilaria Rinaldi, Jean-Michel Létang, Simon Rit

\section{To cite this version:}

Feriel Khellaf, Nils Krah, Ilaria Rinaldi, Jean-Michel Létang, Simon Rit. Effects of transverse heterogeneities on the most likely path of protons. Physics in Medicine and Biology, 2019, 64, pp.065003. 10.1088/1361-6560/ab02a8 . hal-02023848

\section{HAL Id: hal-02023848 \\ https://hal.science/hal-02023848}

Submitted on 12 Oct 2020

HAL is a multi-disciplinary open access archive for the deposit and dissemination of scientific research documents, whether they are published or not. The documents may come from teaching and research institutions in France or abroad, or from public or private research centers.
L'archive ouverte pluridisciplinaire HAL, est destinée au dépôt et à la diffusion de documents scientifiques de niveau recherche, publiés ou non, émanant des établissements d'enseignement et de recherche français ou étrangers, des laboratoires publics ou privés. 


\title{
Effects of transverse heterogeneities on the most likely path of protons
}

\author{
F. Khellaf ${ }^{1}$, N. Krah ${ }^{1}$, I. Rinaldi' ${ }^{2}$ J. M. Létang ${ }^{1}$, S. Rit ${ }^{1}$ \\ ${ }^{1}$ University of Lyon, INSA-Lyon, Université Claude Bernard Lyon 1, UJM-Saint Etienne, CNRS, Inserm, CREATIS UMR \\ 5220, U1206, F-69373, LYON, France. \\ ${ }^{2}$ University of Lyon, Université Claude Bernard Lyon 1, CNRS-IN2P3, Institut de Physique Nucléaire de Lyon, UMR 5822, \\ F-69622, Villeurbanne, France. \\ feriel.khellaf@creatis.insa-lyon.fr
}

\begin{abstract}
The use of a most likely path (MLP) formalism for protons to account for the effects of multiple Coulomb scattering has improved the spatial resolution in proton computed tomography ( $\mathrm{pCT})$. However, this formalism assumes a homogeneous medium and a continuous scattering of protons. In this paper, we quantify the path prediction error induced by transverse heterogeneities to assess whether correcting for such errors might improve the spatial resolution of pCT. To this end, we have tracked protons trajectories using Monte Carlo simulations in several phantoms with different heterogeneities. Our results show that transverse heterogeneities induce non Gaussian spatial distributions leading to errors in the prediction of the MLP, reaching $0.4 \mathrm{~mm}$ in a $20 \mathrm{~cm}$ wide simulated heterogeneity and $0.13 \mathrm{~mm}$ in a realistic phantom. It was also shown that when the spatial distributions have more than one peak, a most likely path, if any, has yet to be defined. Transverse heterogeneities also affect energy profiles, which could explain some of the artifacts described in other works and could make the energy cuts usually performed to exclude nuclear events less efficient.
\end{abstract}

\section{INTRODUCTION}

Proton computed tomography ( $\mathrm{pCT}$ ) has been shown to be a suitable imaging modality to complement or substitute x-ray CT for proton therapy treatment planning. By using the same particles for imaging and treatment, the reconstructed proton stopping power map is more accurate as no conversion is needed from Hounsfield units to relative stopping power (RSP) (Schaffner et al., 1998; Yang et al., 2012; Arbor et al., 2015). This could allow for a reduction of the safety margins used to account for the uncertainty in the proton range (Paganetti, 2012).

However, due to Multiple Coulomb Scattering (MCS), protons go through multiple small angular deflections that lead to a limited spatial resolution in pCT. A compact most likely path (MLP) formalism has been introduced by Schulte et al. (2008) to model the effects of MCS on the proton trajectory. The use of the MLP in pCT reconstruction has significantly improved the spatial resolution (Li et al., 2006; Wang et al., 2011; Rit et al., 2013).

This MLP formalism assumes a homogeneous material which is approximative for several reasons. First, the radiation length of the material $X_{0}$ is needed to compute scattering matrices. The assigned value is $X_{0}=36.1 \mathrm{~cm}$ (Svensson et al., 1984) under the assumption of a homogeneous phantom composed of water. However, denser tissues tend to have a smaller $X_{0}$, e.g. $X_{0}=14.4 \mathrm{~cm}$ for bone or $X_{0}=34.9 \mathrm{~cm}$ for skeletal muscle (Svensson et al., 1984), and therefore scatter protons with larger angles. Furthermore, in order to calculate the variances and covariances of the position and angle of the protons, an evaluation of the ratio $1 / \beta^{2} p^{2}$ is required, where $\beta$ is the proton's velocity relative to the speed of light and $p$ is its momentum, and both depend on the proton energy. This ratio is usually calculated approximately using the energy loss a proton would undergo in a homogeneous phantom (Williams, 2004).

A further approximation is made in the MLP formalism: the calculation of the most likely path is based on the generalized Fermi-Eyges theory (Eyges, 1948) of MCS that uses a Gaussian 
approximation to model the scattering angle and position distributions. In reality, Rutherfordlike scattering events and nuclear interactions (elastic and nonelastic) both induce large-angle scattering causing non-Gaussian tails in the distributions. The former effect is negligible for the purpose of proton imaging and the latter is usually mitigated by applying angle and energy cuts which allow to exclude most protons that have experienced these interactions (Schulte et al., 2008). The Gaussian approximation is therefore valid with appropriate cuts as long as tissue properties change only as a function of depth (panel A or B in Figure 2), but not along a direction perpendicular to the beam axis. In the case of a transverse heterogeneity (a heterogeneity with a finite lateral dimension that would cause an unbalanced scattering of protons, see panel $\mathrm{C}$ in Figure 2), the distribution is expected to be asymmetric as the scattering would differ depending on the lateral coordinate. Such a spatial asymmetry of the MCS in a heterogeneous phantom cannot be corrected simply by introducing information about the tissues' density and composition in the current formalism, but would require a reformulation of the MLP formalism itself. If the heterogeneity is large enough, this could cause a systematic error on the path prediction that would be inherent to the formalism, as the MLP calculations are based on symmetric distributions.

We observed the effect of unbalanced scattering around transverse heterogeneities experimentally: proton beam profiles acquired with a pair of strip detectors (Krah et al., 2018) at the Curie Institute - Proton Therapy Center in Orsay, France, showed non Gaussian distributions when the protons traversed interfaces (e.g. water/bone or water/lung) of the CIRS electron density phantom. These profiles, as well as a map representing their skewness, i.e. their asymmetry, are shown in Figure 1. The edges of the inserts of bone and lung in the water phantom are associated with either high or low skewness values, depending on the orientation of the interface. It is not the purpose of this work to quantitatively analyse the measured beam profiles. They serve as illustrative evidence of the effect of material heterogeneities on MCS.

Similar observations of unbalanced scattering were made by West et al. (1972) and West et al. (1973) who noticed that a difference in the scattering of protons around edges normal to the beam would cause fringes in the intensity distribution (this was called the "West-Sherwood effect" by Cormack et al. (1976)). While West and Sherwood saw this effect as a way to better distinguish the edges of an object in radiography, it is presented as a cause of deterioration of image quality in the pCT system described by Tanaka et al. (2016) and Tanaka et al. (2018). In this case, the West-Sherwood effect at the edges causes an underestimation of all pixel values inside the object. Finally, Zhang et al. (2017) observed the effects of transverse heterogeneities in the form of range-mixing, i.e. a combination of different dose rate functions around an interface, causing a loss of spatial resolution. These observations were made in the context of proton radiography or tomography, using different kinds of set-ups. We specifically explore the effects of unbalanced scattering in single tracking in our work.

Some studies have been conducted on heterogeneities in the MLP framework. Wong et al. (2009) have studied the impact of longitudinal heterogeneities (tissue variations as a function of depth) on the MLP. They have used the mean error in addition to the root mean square error (RMS). Their results showed no significant systematic error caused by longitudinal heterogenenities. This had been predicted by Williams (2004), where it was expected that changes in density would have little effect, except for a proton trajectory grazing the surface of a large object with a small dimension perpendicular to the beam, e.g. a bone plate.

More recently, Collins-Fekete et al. (2017) have extended the MLP formalism by including prior knowledge on the medium composition and density. The proposed method is based on an MLP formalism that only models MCS for longitudinal heterogeneities, without taking into account transverse ones. Furthermore, their evaluation with the RMS does not reflect the MLP accuracy, as demonstrated by Wong et al. (2009), where longitudinal heterogeneities caused an increase in RMS but no change in MLP accuracy measured by the mean error. Unlike CollinsFekete et al. (2017), we have not compared different models to estimate the MLP but have 
focused on Monte Carlo proton tracks to determine the MLP and evaluated the accuracy of the conventionally used MLP formalism of Schulte et al. (2008).

In this paper, we investigate the effects of tissue heterogeneities, and particularly transverse heterogeneities, on the proton path prediction. Specifically, we study the kind and degree of deflection due to transverse heterogeneities and its impact on the energy spectra of the protons.
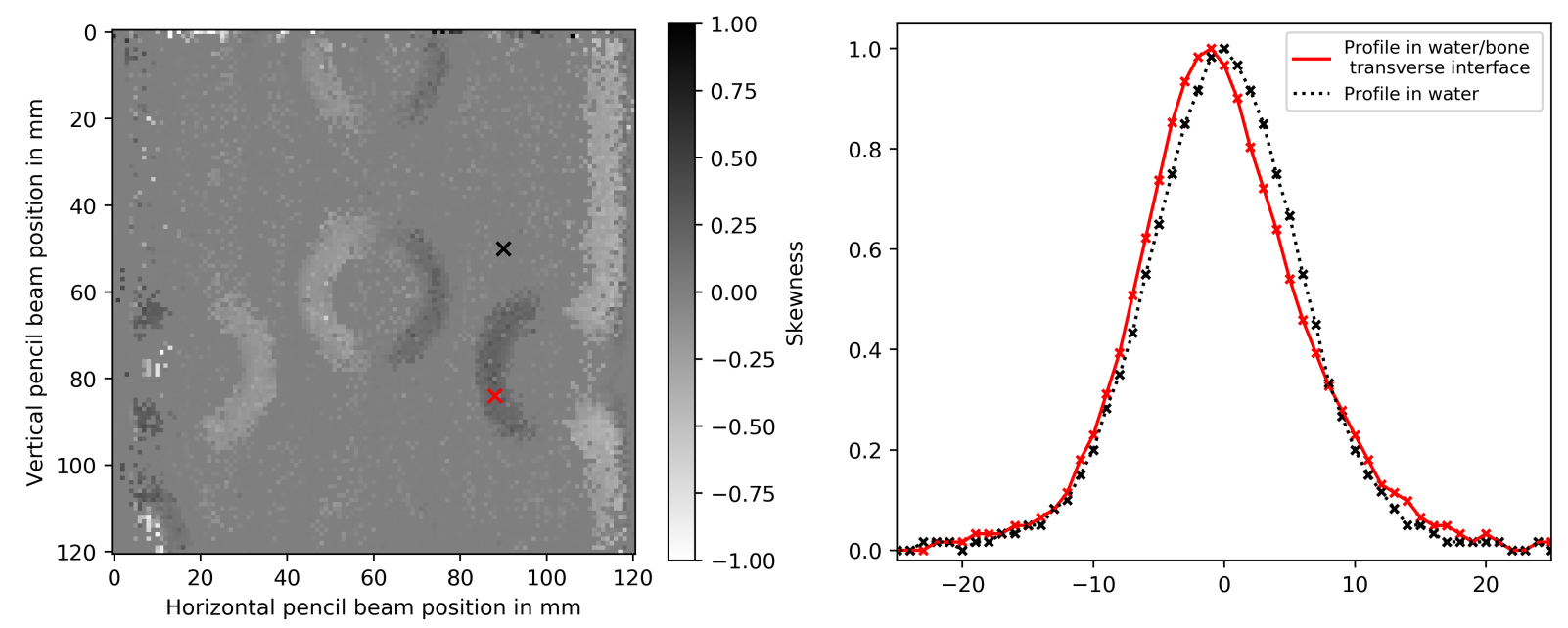

Figure 1: 2D map of the skewness of each beam profile in a CIRS phantom with inserts (left) and fluence profiles along the horizontal position measured at a water/bone interface (right). Crosses in left image indicate the pencil beam position for which the profile is shown.

\section{MATERIALS AND METHODS}

\subsection{Theoretical MLP}

In what follows, the term "theoretical MLP" refers to the approximate MLP calculated with the formalism of Schulte et al. (2008) and "real MLP" means the MLP derived from the Monte Carlo (MC) results. The most likely path of protons described by Schulte et al. (2008) is computed as follows:

$$
y_{\mathrm{MLP}, \text { theo }}(u)=\left(\Sigma_{1}^{-1}+R_{1}^{T} \Sigma_{2}^{-1} R_{1}\right)^{-1}\left(\Sigma_{1}^{-1} R_{0} y_{0}+R_{1}^{T} \Sigma_{2}^{-1} y_{2}\right)
$$

where $u$ is the depth in the phantom, $y_{0}$ and $y_{2}$ are vectors containing the entry and exit coordinates (position $t$ and angle $\theta$ ), $\Sigma_{1}$ and $\Sigma_{2}$ are the scattering matrices, and $R_{0}$ and $R_{1}$ are essentially rotation matrices. The elements of the scattering matrices are given by the variances and covariances of the position and angle coordinates defined in Equations (7) to (9) and (16) to (18) in (Schulte et al., 2008). In addition, the MLP uncertainty matrix is given by

$$
\varepsilon(u)=\left(\Sigma_{1}^{-1}+R_{1}^{T} \Sigma_{2}^{-1} R_{1}\right)^{-1}
$$

where the uncertainty on the lateral displacement is given by the element in the first row and first column of $\varepsilon(u)$. As mentioned in the introduction, the radiation length $X_{0}$ and momentumvelocity ratio $1 / \beta^{2} p^{2}$ are calculated using a homogeneous water medium assumption.

A theoretical MLP taking into account a depth dependent heterogeneity can be computed using Equation (4) in (Collins-Fekete et al., 2017) to find the scattering matrices. Unlike CollinsFekete et al, we used $E_{0}=13.6 \mathrm{MeV}$ instead of 14.1 and the constant 0.038 instead of $1 / 9$ as recommended in (Schulte et al., 2008). This was done for a more homogeneous comparison with Schulte's formalism. The matrices were computed using the radiation length and the momentum-velocity ratio based on the known geometry of heterogeneous phantom (B). 


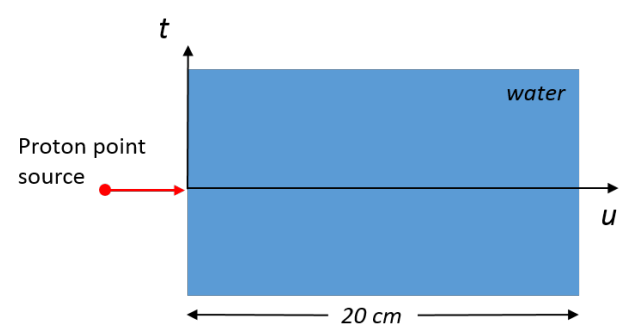

(A)

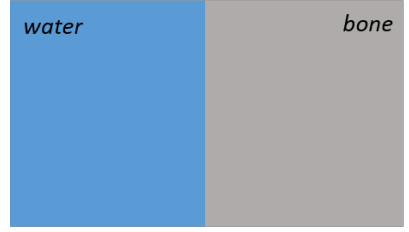

(B)

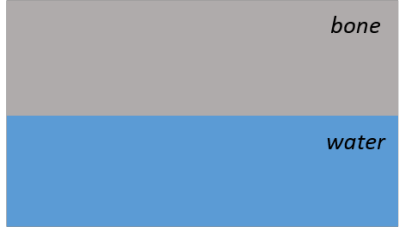

(C)

Figure 2: Phantoms used in the simulations. The blue material is water and the grey one is bone.

\subsection{Monte Carlo simulations}

We have used GATE v8.0 (Jan et al., 2011), a software based on Geant4 v10.3.2 (Agostinelli et al., 2003), to simulate proton paths in different media: three very basic phantoms to study the effects of transverse heterogeneities in simple conditions and one anthropomorphic head phantom. First, we have studied the three phantoms shown in Figure 2; a homogeneous water phantom (A), a phantom including a longitudinal heterogeneity where protons go through the interface between water and bone (B), and a phantom with a transverse heterogeneity such that the protons are grazing the interface $(\mathrm{C})$. Although these phantoms exaggerate heterogeneities in patients, we used them to quantify the maximum deviation of the MLP from the one estimated with the conventional formalism that could occur in such an extreme scenario. Second, to quantify the error on the MLP in a more realistic case, we have used an ICRP head phantom (ICRP, 2009). We automatically identified the regions with the largest heterogeneities by computing the projections of the derivative along the cranio-caudal axis of the $1 / X_{0} 3 \mathrm{D}$ map and locating its maximum values.

We selected two transverse regions : the interface between air $\left(X_{0}=6.3 \times 10^{11} \mathrm{~cm}\right)$ and the top of the head $\left(X_{0}=43.4 \mathrm{~cm}\right)$ which was about $3.4 \mathrm{~cm}$ long, and the one between the teeth $\left(X_{0}=9.0 \mathrm{~cm}\right)$ and the mandible $\left(X_{0}=29.4 \mathrm{~cm}\right)$ which was about $7.7 \mathrm{~cm}$ long. For the first heterogeneity, the beam was positioned at $t=803.4 \mathrm{~mm}$ and for the second one it was at $t=634.0 \mathrm{~mm}$ (see Figure 8). The voxel spacing along the vertical axis was of $4.84 \mathrm{~mm}$. In addition to the tracking inside the phantom, two ideal detectors were positioned $40 \mathrm{~cm}$ upstream and downstream from the isocenter. All targets (phantoms (A), (B), (C) and ICRP phantom) were surrounded by vacuum.

A $250 \mathrm{MeV}$ monoenergetic proton source was simluated to traverse these phantoms, and a parallel beam geometry was used to keep the protons tangent to the transverse interface. For the three phantoms in Figure 2, an infinitely narrow point source was used at different entry positions (see section 2.3). For the ICRP phantom, we simulated a uniform rectangular $4 \times 4$ $\mathrm{mm}$ proton source. $10^{7}$ protons were simulated for each tested path and for each particle the trajectory was tracked inside the phantom using a maximum step size of $5 \mathrm{~mm}$ (the actual step size is smaller, $1.4 \mathrm{~mm}$ on average in the ICRP phantom presented below since it is limited by the voxel geometry). The emstandard physics list was used to simulate only electromagnetic processes, as nuclear interactions are not accounted for in the MLP formalism. Simulations using Geant4's QGSP_BIC physics list were also performed to assess the impact of nuclear interactions. The protons' kinetic energy was recorded since an asymmetry in the spatial and angular distributions would also affect the energy distribution.

\subsection{Post-processing}

To compute the real MLP of protons going through each phantom, we first selected the trajectories of a subset of protons whose entry and exit coordinates, $y_{0}$ and $y_{2}$, lay within a small 


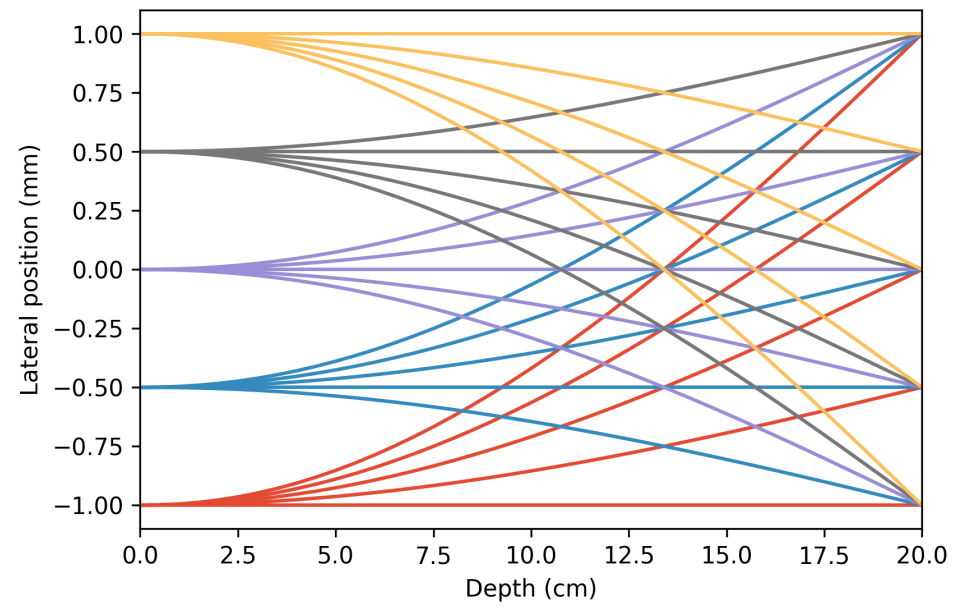

Figure 3: Theoretical MLPs for all tested entry and exit coordinates. Different colors were used depending on the entry position for a clear visualization. The exit angles $\theta_{2}$ are equal to $0, \pm 4$, $\pm 8, \pm 12$, and $\pm 16 \mathrm{mrad}$ for lateral deviations $\Delta t$ equal to $0, \pm 0.5, \pm 1.0, \pm 1.5$, and $\pm 2.0 \mathrm{~mm}$, respectively. The axes' scales are different for a better visualization.

interval around chosen values, $t_{1}, \theta_{1}, t_{2}$ and $\theta_{2}$. Specifically, we filtered out those whose angle and position would be experimentally indistinguishable by filtering the protons using windows of size $200 \mu \mathrm{m}$ for the position and $3.4 \mathrm{mrad}$ for the angle. These thresholds reflect the typical spatial and angular resolution of detectors used in pCT (Bopp et al., 2014). Since the proton sources were simulated without angular deviation, the entry angle was not filtered. In addition, for the three test phantoms (A), (B) and (C), there was no need to filter the entrance position as we used a point source. We then determined the real MLP of protons with such entry and exit coordinates by locating the most probable position at each depth in the phantom.

On the other hand, we calculated the theoretical MLP using the formalism of Schulte et al. (2008). As entry coordinate, we used the parameters from the point source definition for phantoms (A), (B) and (C) and the center of the filtering window for the anthropomorphic phantom. For the exit coordinates, we used the center of the filtering windows.

We have tested several combinations of entry and exit coordinates to find the maximum bias created by heterogeneities. In the particular case where $y_{0}$ and $y_{2}$ are equal, the theoretical MLP is a straight line. We varied the values of $t_{0}$ and $t_{2}$, while keeping $\theta_{0}$ zero. For $\theta_{2}$, we took the most probable angle corresponding to each $t_{2}$ calculated using the correlation coefficient in the scattering matrix in the case of homogeneous water. The therotical MLPs for the tested entry and exit coordinates are shown in Figure 3 . For each case, we compared the theoretical MLP to the real MLP.

To estimate the real MLP, we have built a histogram of the transverse positions at each depth in the phantom and fitted a two-term Gaussian distribution to it:

$$
f(x)=\frac{A_{1}}{\sigma_{1} \sqrt{2 \pi}} e^{-\frac{\left(x-\mu_{1}\right)^{2}}{2 \sigma_{1}^{2}}}+\frac{A_{2}}{\sigma_{2} \sqrt{2 \pi}} e^{-\frac{\left(x-\mu_{2}\right)^{2}}{2 \sigma_{2}^{2}}} .
$$

We found that such a two-term Gaussian was more suitable than a single normal distribution since the distribution could be asymmetric in the case of transverse heterogeneities. The distributions were filtered using a Gaussian of $\sigma=3 \times 10^{-2} \mathrm{~mm}$ before fitting, as the two-term Gaussian could be sensitive to noise, especially in the cases where a simple Gaussian would have sufficed (homogeneous and longitudinally heterogeneous cases). We determined the real MLP at a depth $u, y_{\text {MLP,real }}(u)$, as the "mode", that is the position of the maximum, of the fitted function which was more robust to noise than taking the maximum of the histogram directly. We compared this real MLP to the theoretical MLP computed with Equation 1, and the real 
MLP's uncertainty envelope to the theoretical uncertainty given by Equation 2. The maximum absolute bias defined as

$$
\max _{u}\left|y_{\mathrm{MLP}, \text { real }}(u)-y_{\mathrm{MLP}, \text { theo }}(u)\right|
$$

was used to characterize the accuracy of the theoretical MLP formalism. We estimated the uncertainty envelope of the real MLP using the $16^{\text {th }}$ and $84^{\text {th }}$ percentiles of the fitted distribution. When the distribution is symmetric, this is equivalent to one standard deviation from the mean. Otherwise, we obtain an asymmetric envelope which reflects the skewness of the distribution.

\section{RESULTS}

\subsection{Results for phantoms (A), (B) and (C)}

Figure 4 shows the proton trajectories in the three different phantoms in the case of a theoretical straight line path and an example of the two-term Gaussian fit used to compute the real MLP halfway into the phantom. The most likely path computed from the most probable position at each depth does not differ from the theoretical MLP in case of a homogeneous phantom and a phantom with a longitudinal heterogeneity. For the transverse heterogeneity, the most likely path is deflected towards the water part of the phantom. In this case, the deviation reaches about $-0.28 \mathrm{~mm}$, but is still of the same magnitude as the uncertainty envelope. The uncertainty envelope of the real MLP is asymmetric with the larger side of the envelope situated towards the bone part of the phantom. For phantom (A), the uncertainty envelope closely follows the theoretical envelope and for phantom (B), it is wider in the bone region. For both phantoms (A) and (B), the distribution is symmetric and a simple Gaussian would have been sufficient to fit the data. For phantom (C), the two-term Gaussian is not a perfect fit, but works well enough to locate the most probable position. Also, we note that using the fit to find the maximum was more robust than extracting it from the data directly as the data was quite noisy due to the filtering of the trajectories. The cuts on the exit coordinates filter out more than $99 \%$ of protons. More precisely, we kept approximately 30000 protons of the original $10^{7}$ simulated protons for phantom (A), 15000 for phantom (B) and 16000 for phantom (C). The number of filtered protons is lower in case of heterogeneous phantoms as materials with a higher density - in our case, bone - scatter protons with larger angles.

The upper plots of Figure 5 represent the exit spatial and angular distributions of protons $20 \mathrm{~cm}$ downstream of the three phantoms without any filtering. The distributions are Gaussian for both phantoms (A) and (B), although the peak is more spread out for the heterogeneous phantom as protons traversing bone are scattered with larger angles. The position and angle distributions for phantom (C) are not symmetric, with peak positions at $y_{\text {peak }}=-3.8 \mathrm{~mm}$ and $\theta_{\text {peak }}=-12.5 \mathrm{mrad}$, respectively, meaning the protons are scattered primarily towards the less dense material i.e. water. The asymmetry of the spatial distribution of phantom (C) explains what has been observed in the real data presented in Figure 1. The distributions including nuclear interactions are close to the profiles with electromagnetic (EM) interactions only. The difference lies in the presence of large angle MCS tails due to nuclear collisions, but the position of the maximum, i.e. the most probable position is the same.

In Figure 6, the RMS error for the same subset of protons used in Figure 4 (protons filtered with $\left.y_{0}=y_{2}=(0,0)\right)$ is shown. The error was estimated using the homogeneous formalism and the heteregeneous formalism presented in (Collins-Fekete et al., 2017). The maximum RMS, calculated using the homogeneous formalism, reaches $0.51 \mathrm{~mm}$ for phantom (B) and $0.46 \mathrm{~mm}$ for phantom (C), compared to $0.34 \mathrm{~mm}$ for the homogeneous phantom. For both phantoms (B) and (C), the RMS error calculated using the heterogeneous formalism is almost identical to the one assuming a water medium. For the longitudinal heterogeneity, the maximum is shifted towards the bone region. 

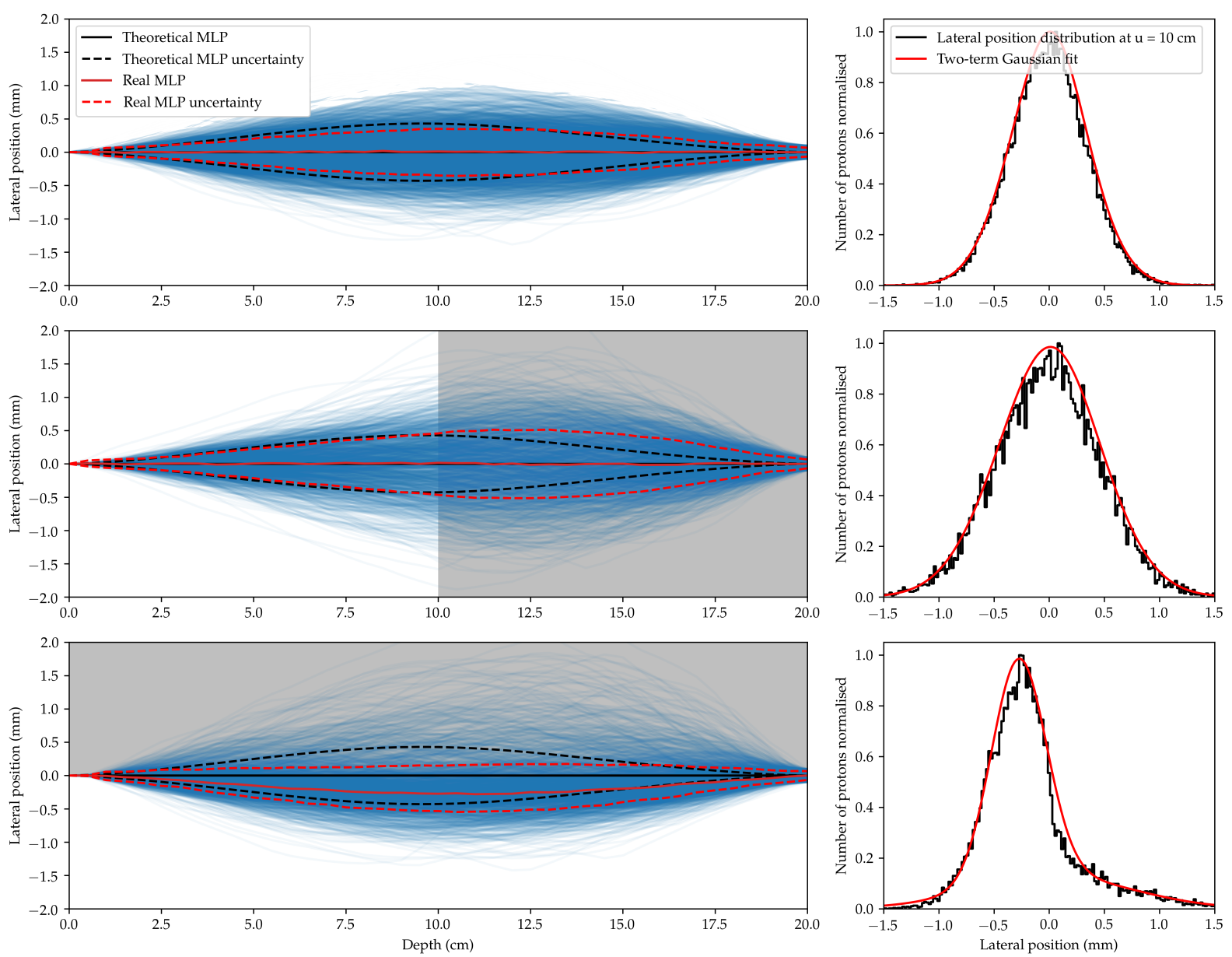

Figure 4: Trajectories of filtered protons (left) going through phantoms A (top), B (middle) and $\mathrm{C}$ (bottom) and an example fit used to compute the real MLP (right). The shaded grey areas correspond to bone. 

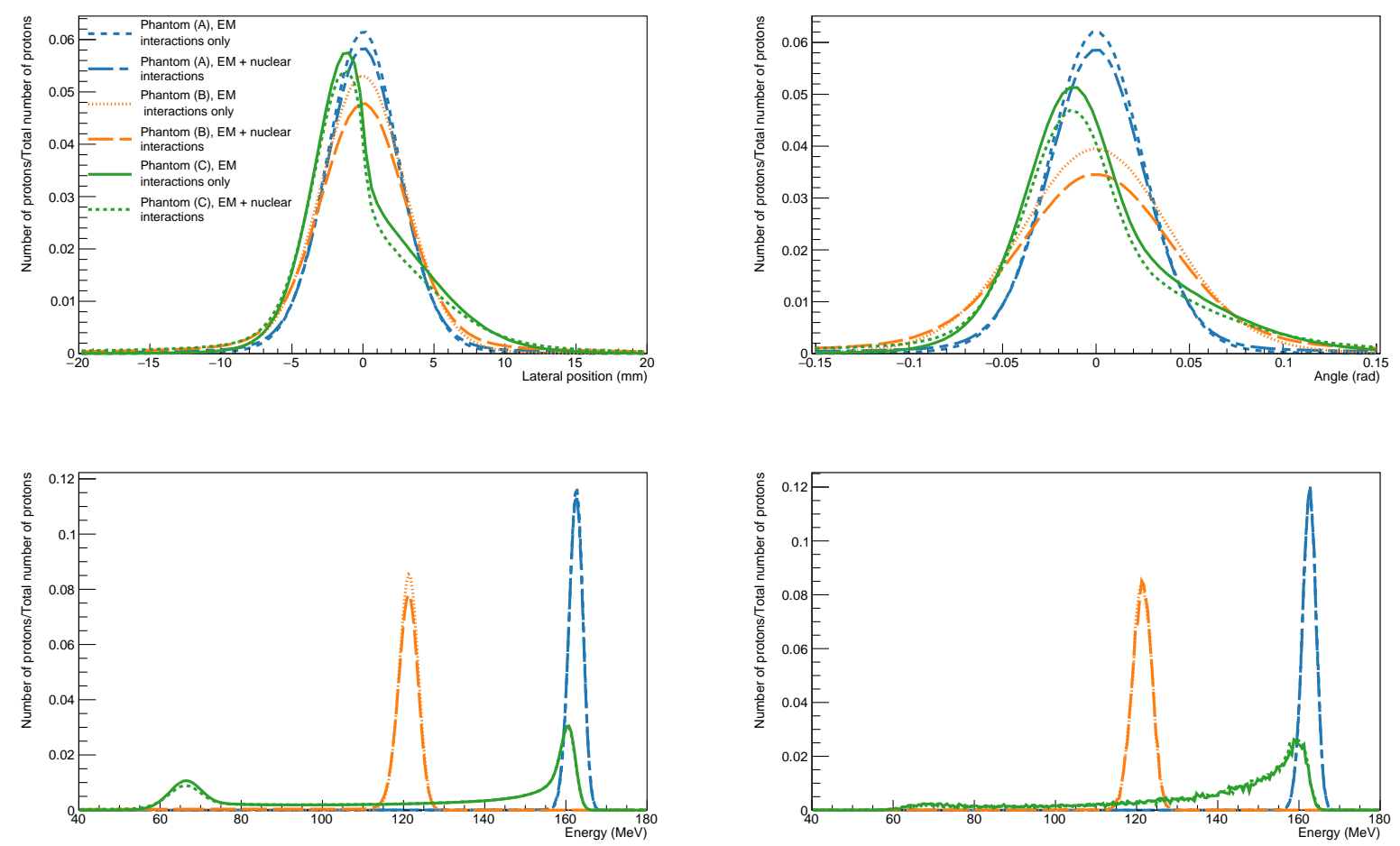

Figure 5: Exit spatial (top left) and angular (top right) distributions and exit energy distribution of unfiltered protons (bottom left) and filtered protons with $y_{0}=y_{2}=(0,0)$ (bottom right) .

In Figure 7, we show the real and theoretical MLP for different exit coordinates in the three phantoms. For homogeneous phantom (A), the MLP formalism works very well as there is no large difference between the theoretical and real MLP. Phantom (B) displays an increasing bias when the path diverges from a straight line, although this error is smaller than the bias measured in the case of transverse heterogeneities. Using an adapted MLP formalism for a longitudinally heterogeneous phantom importantly reduces the bias which reaches similar levels as the error in the homogeneous phantom. Finally, for phantom (C), the bias is largest for protons exiting the phantom from the bone region (positive exit positions), with a maximum bias of $0.41 \mathrm{~mm}$ is for $t=1 \mathrm{~mm}$.

The lower plots of Figure 5 show the energy distributions of unfiltered and filtered protons. For phantoms (A) and (B), the distributions are similar except that protons have traversed

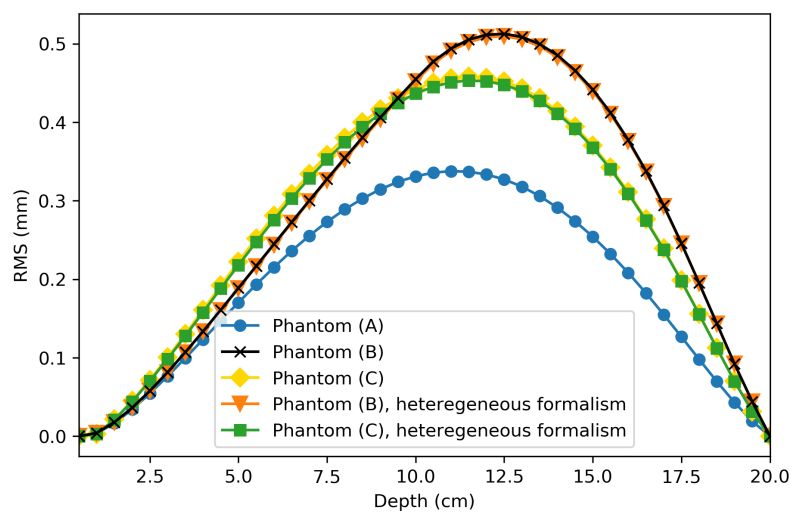

Figure 6: RMS error for the three phantoms. 

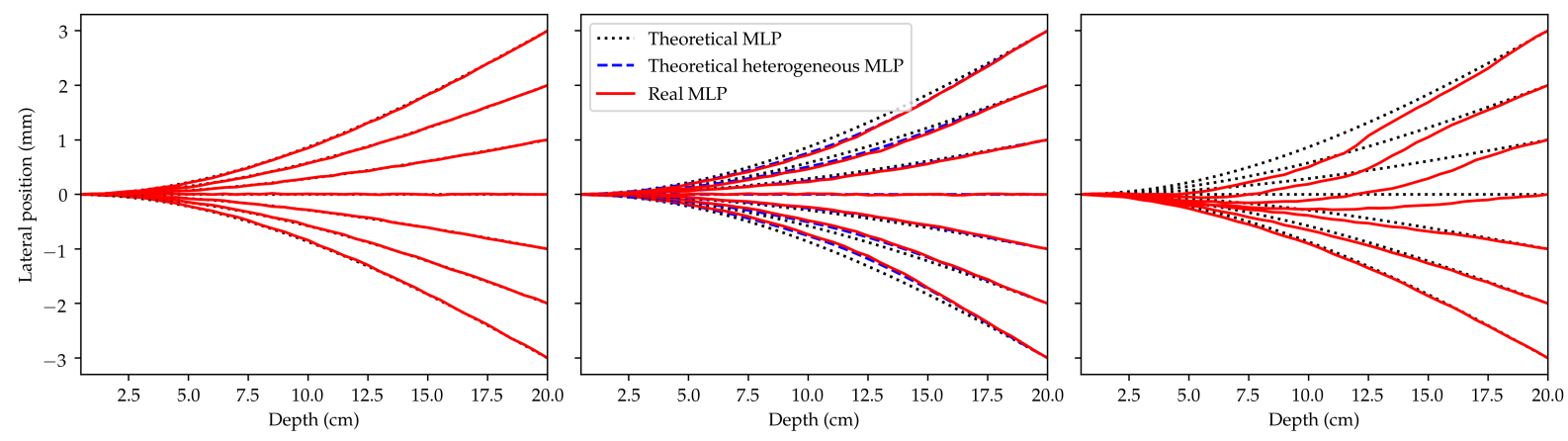

(a)

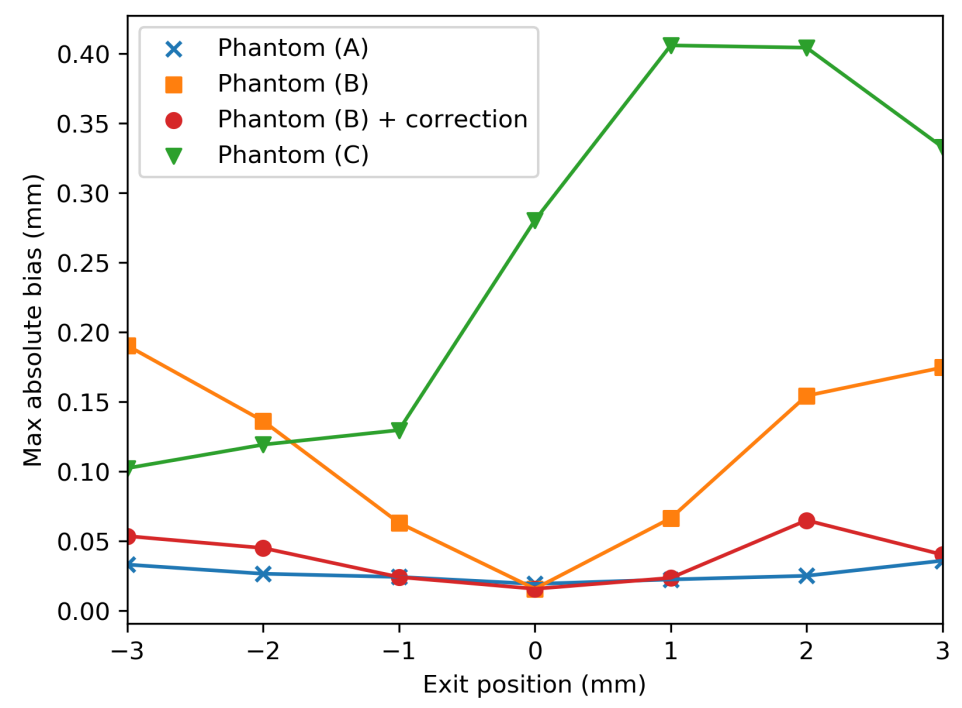

(b)

Figure 7: Comparison of the theoretical and real MLP for different exit positions : (a) plot of the paths in the phantoms (A), (B) and (C), from left to right, (b) maximum absolute difference between real and theoretical paths. For phantom (B), the theoretical MLP computed using ground truth knowledge of the tissues was additionally computed. 
bone in case of phantom (B) and have consequently lost more energy. The peak energy is $163 \mathrm{MeV}$ for phantom (A) and $121 \mathrm{MeV}$ for phantom (B). Also, their distributions with and without filtering the coordinates are very much alike. As for phantom $(\mathrm{C})$, the distribution for unfiltered protons shows two peaks at $161 \mathrm{MeV}$ and $66 \mathrm{MeV}$, corresponding to protons that traversed mostly water and bone, respectively. The amplitude of the high energy peak is three times greater than the low energy peak's amplitude, thus confirming more protons have passed through the water region. As for the distribution of filtered protons, the low energy peak is barely distinguishable, as almost all protons have traveled the water rather than the bone material. In addition, for both distributions, the plateau region between the two peaks shows that a lot of protons have an intermediate energy after having traversed both materials. The difference between the distributions with and without nuclear interactions is barely distinguishable for both unfiltered and filtered data.

\subsection{Results for ICRP phantom}

Figure 8 shows the Monte Carlo trajectories of the protons along the two selected transverse heterogeneities with coordinates $y_{0}$ and $y_{2}$ centered on the interface. For the first interface between air and head, the spatial distribution is clearly affected by this heterogeneity: the majority of protons pass through air, where they are hardly scattered. We note that the entry and exit coordinates for the real and theoretical MLP are different : although the original beam's distribution is uniform, the filter only keeps protons having a straight trajectory (since $y_{0}=y_{2}$ ), thus selecting a majority of protons that have travelled through air. Regarding the real MLP, it follows a quasi linear path in the air region then gets closer to the theoretical MLP as protons scattered in the head region pass in the air region. A maximum bias of $0.085 \mathrm{~mm}$ is reached around the entry and exit of the phantom. The spatial distribution for the second heterogeneity resembles the one observed for Phantom (C) (a slightly skewed Gaussian). The maximum bias between the theoretical and real MLP was measured at a depth of $-34 \mathrm{~mm}$ and reached $0.075 \mathrm{~mm}$.

The right column of Figure 8 shows the energy distributions for the same subset of protons on the exit detector for the two interfaces. Regarding the air/head interface, practically all protons exit with an energy of $250 \mathrm{MeV}$ as the majority has passed in the air region. Regarding the interface between teeth and mandible, the energy profile shows one major peak at $200 \mathrm{MeV}$ and a smaller one at $185 \mathrm{MeV}$.

\subsection{Impact of entry and exit positions}

We first focused on phantom (C) and examined the impact of different entry and exit positions on the MLP. The spatial exit distributions as well as the energy profiles for beams starting at $t_{0}=-1,-0.5,0,0.5,1 \mathrm{~mm}$ are shown in Figure 9. The beams starting in the bone region (at $t_{0}=0.5 \mathrm{~mm}$ and $1 \mathrm{~mm}$ ) clearly show a non Gaussian bimodal distribution, that is a distribution with two peaks. In these cases, a two-term Gaussian is a better fit, therefore legitimating its use to find the real MLP. The other distributions have a single maximum, but are distorted compared to a single Gaussian.

The characteristic shape of the spatial distributions of protons starting in the bone region bone is due to their trajectory shown in Figure 10a. The trajectories of protons starting at $t_{0}=1 \mathrm{~mm}$ are traced and each path is colored depending on the percentage of trajectory the proton has spent in bone. There is a separation between protons spending more than $95 \%$ of their trajectory in the bone region and the rest of protons, meaning most protons which exit the phantom in the bone region have spent their whole trajectory in bone while protons who have spent part of their trajectory in the water region stay in water. This is clearly depicted in Figure 10b showing the histograms of the percentage of trajectory spent in bone for protons exiting in the bone or water region. More precisely, $54 \%$ of protons did not traverse the water 

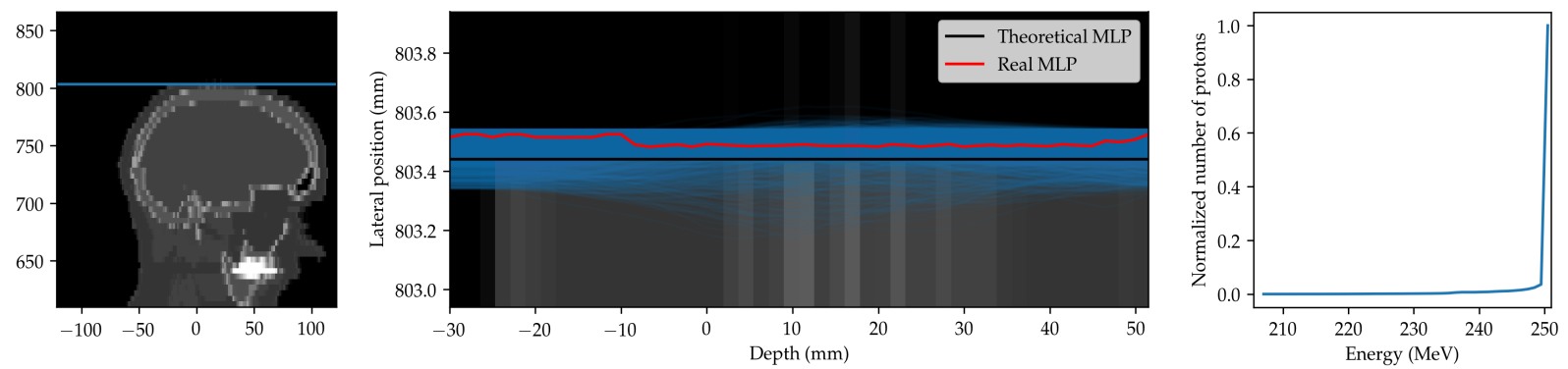

(a)
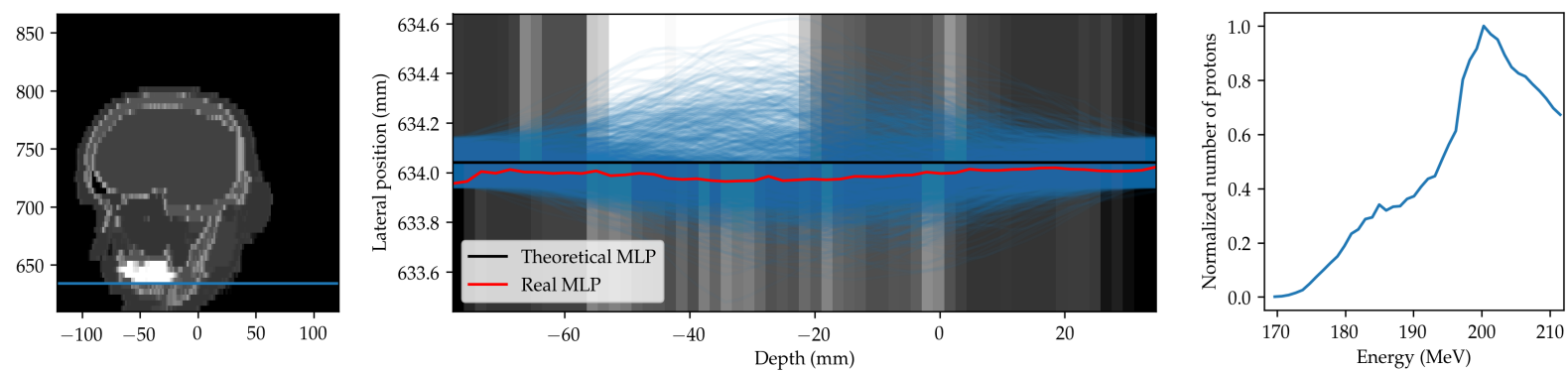

(b)

Figure 8: Proton trajectories along two transverse heterogeneities. On the left, the beam position is shown on the $1 / X_{0}$ map, in the middle, the trajectories are shown on a zoomed area of the same map. Most small longitudinal heterogeneities are just an effect of the spline interpolation done to rotate the image for visualization. On the right are represented the energy distributions on the exit detector.

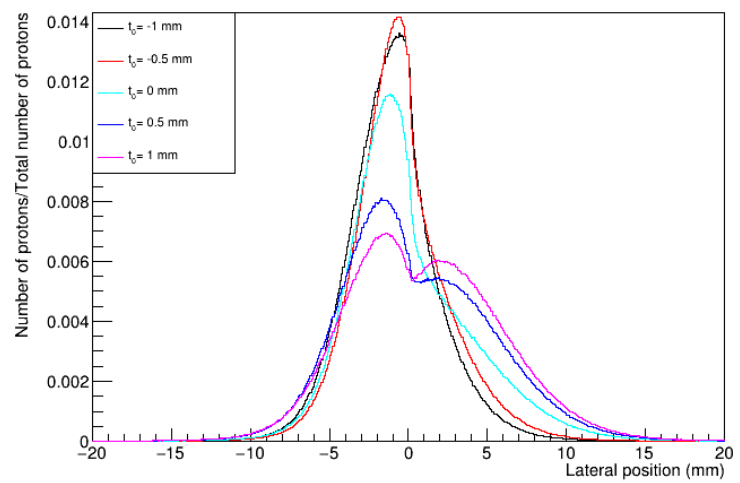

(a)

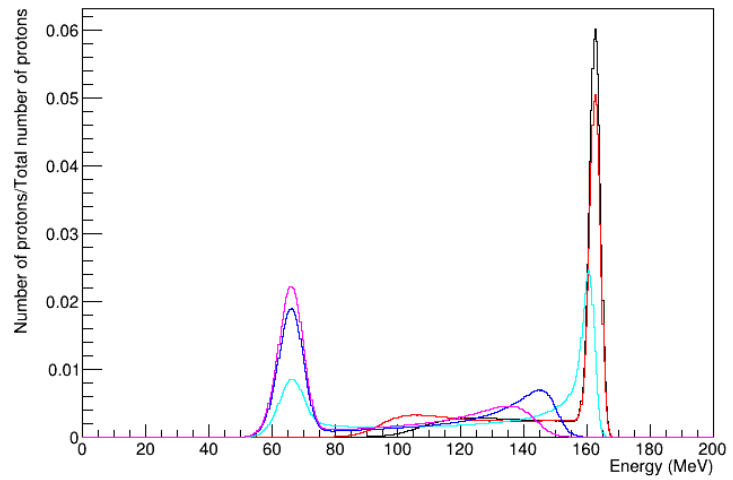

(b)

Figure 9: Exit spatial (a) and energy (b) distributions for beams starting at different positions, without any selection on the exit position. Negative $t$ values are in water, positive ones are in bone. 


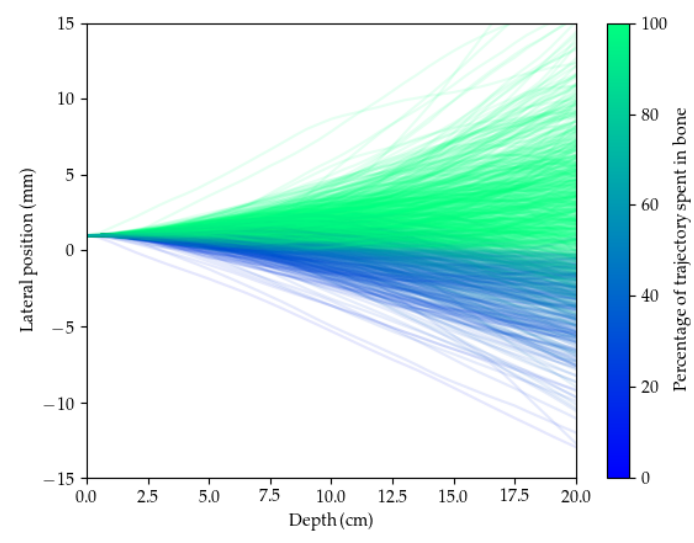

(a)

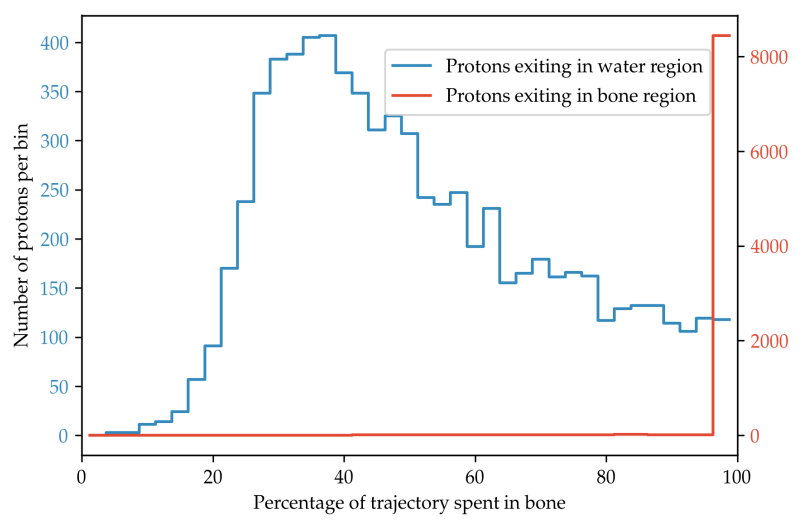

(b)

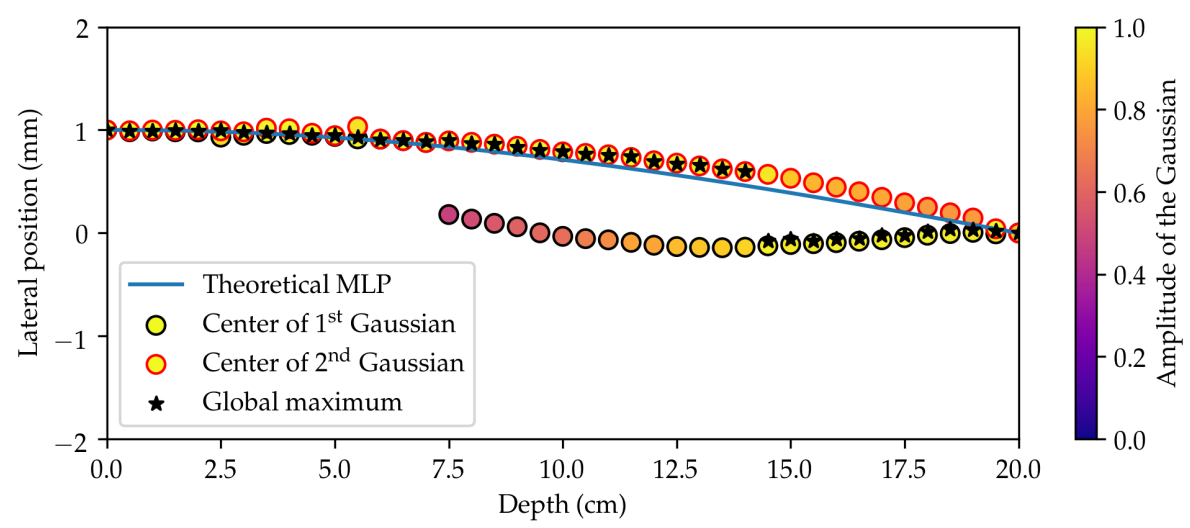

(c)

Figure 10: (a) Unfiltered trajectories for beam starting at $t_{0}=1 \mathrm{~mm}$, each trajectory is colored according to the time the proton has spent in the bone region (a value of zero means the proton has spent all its trajectory in water and a value of 100 corresponds to a proton that has exclusively passed in bone). Negative lateral positions are in water, positive ones are in bone. (b) Histogram of the percentage of trajectoy spent in bone for protons exiting in bone (red), or water region (blue). (c) Proton path starting at $t_{0}=1 \mathrm{~mm}$ and exiting at $t_{2}=0 \mathrm{~mm}$ : the real MLP defined by the most probable position at each depth is discontinuous.

region at all and $44 \%$ have made a single transition from bone to water. Figure 9(b) confirms the observations made above. For proton beams starting in bone, there is a first peak in bone corresponding to protons that have stayed in the initial medium; and a second smaller peak corresponding to protons that have passed to the adjacent water medium.

Figure 10c shows an example where defining an MLP is challenging. This issue arises when there is more than one peak in the spatial distributions (Figure 9a). The figure shows that the major mode of the distribution (the position of the global maximum), which has been used to represent the MLP in previous works, suddenly jumps from 0.6 to $-0.1 \mathrm{~mm}$ at $u=14 \mathrm{~cm}$. The parameters $\mu_{1}$ and $\mu_{2}$ of each Gaussian are also displayed, as well as their amplitude. At first, the distribution is normal and the two Gaussians share the same center. Then, this first Gaussian distribution declines while a second Gaussian appears at $u=7.5 \mathrm{~cm}$, as more and more protons transition to the water region. At $u=14 \mathrm{~cm}$, this second Gaussian becomes predominant. The path obtained using the most probable position at each depth is discontinuous, while the one following the center of the first Gaussian stays close to the theoretical MLP.

Figure 11a shows the MLP bias in phantom (C) for various entry and exit positions. Roughly speaking, protons with entry and exit coordinates in the same medium (top-left and bottom- 
right quarters) have biases that are lower than protons whose entry and exit positions are in different tissues or exactly at the interface between the two media (bottom-left and top-right quarters). In addition, the bias is larger when protons start their path in the denser tissue since they are more likely to transition to a different medium. The maximum bias of $0.5 \mathrm{~mm}$ is found for protons starting in the bone region and exiting at the interface.

Finally, the bias reached for different entry and exit parameters using the ICRP phantom is shown in Figure 11. Regarding the air/head interface, some trajectories involving "large" lateral deviations (i.e. $>=0.5 \mathrm{~mm}$ ), for example $t_{0}=804.5 \mathrm{~mm}$ and $t_{2}=802.5 \mathrm{~mm}$, were not taken by any protons or a very small number of protons because there was not enough scattering. These cases correspond to the hatched regions on the figure. All measured biases, for both heterogeneities, were quite small compared to the ones calculated using phantom $(\mathrm{C})$. The largest bias for the air/head heterogeneity was $0.10 \mathrm{~mm}$ and it reached $0.13 \mathrm{~mm}$ for the teeth/mandible.

\section{DISCUSSION}

We have shown that the Gaussian assumption on the spatial and angular distribution functions used to compute the MLP of protons is not valid when unbalanced scattering occurs due to transverse heterogeneities. When protons traverse two different tissues, the denser material will scatter the particles with larger angles, thus more protons will end up in the less dense material. This causes skewed or even double peaked distributions, with a maximum located in the less dense material, as seen in Figures 5 and $9 \mathrm{a}$.

In terms of MLP, this asymmetry creates a bias between the real MLP obtained with MC simulations and the theoretical MLP obtained under the assumption of a homogeneous object (Schulte et al., 2008). For protons having the same entry and exit coordinates for instance, the theoretical MLP predicts a straight path while the real MLP (in phantom (C), or both heterogeneities in the ICRP phantom) is in fact leaning towards the less dense medium (Figure 4 and Figure 8). We made sure our choice of spatial and angular filter values was small enough to consider the exit position constant and large enough to keep enough protons for our analysis. Dividing the filter thresholds by a factor of 4 did not change our results. We note in Figure 4 that the uncertainty envelopes for the real MLP are larger than the theoretical MLP's envelopes at the end of the trajectory since the variance of the exit coordinate was not taken into account to compute the theoretical MLP and its uncertainty. Other minor differences regarding the error envelope for Phantom (A) are attributed to the difference between the MCS models used in Gate and in the MLP formalism, and the approximate calculation of the $1 / \beta^{2} p^{2}$ ratio. For both phantoms (B) and (C), the envelope is wider in the bone region since protons are more scattered in a dense tissue, leading to an asymmetrical envelope for the transverse phantom. For phantom (C), the maximum deflection of the real MLP compared to the straight line is about $0.28 \mathrm{~mm}$. For other trajectories than the basic straight line path, the maximum bias has been evaluated at $0.5 \mathrm{~mm}$ (Figure 11a). However, it was also shown that in cases when the distribution is not unimodal (when there is more than one peak, as seen when protons start their trajectory in bone in Figure 99, the MLP estimated using the lateral position distribution major mode (the most probable position) may produce a discontinuous trajectory. Therefore, in theses cases, the bias does not reflect the difference between the real MLP and theoretical MLP but rather the difference between the most probable position and the theoretical MLP. If we only consider the cases with unimodal distributions (three first rows of Figure 11a), the maximum bias reaches $0.41 \mathrm{~mm}$. This is of the same order of magnitude as the maximum MLP uncertainty which reaches $0.43 \mathrm{~mm}$ in water. The maximum measured bias for a realistic heterogeneity only reached $0.13 \mathrm{~mm}$ (Figure 11c).

Furthermore, we were able to verify that an adaptation of the formalism including a depthdependant radiation length and momentum-velocity (Collins-Fekete et al., 2017) is sufficient to 


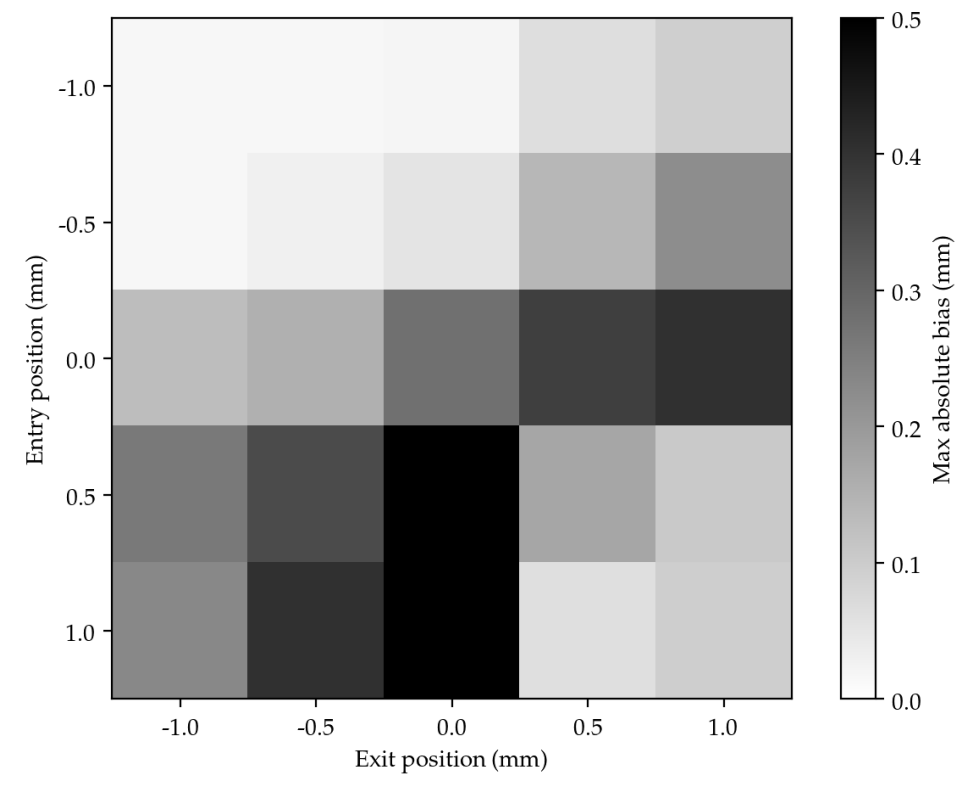

(a) a

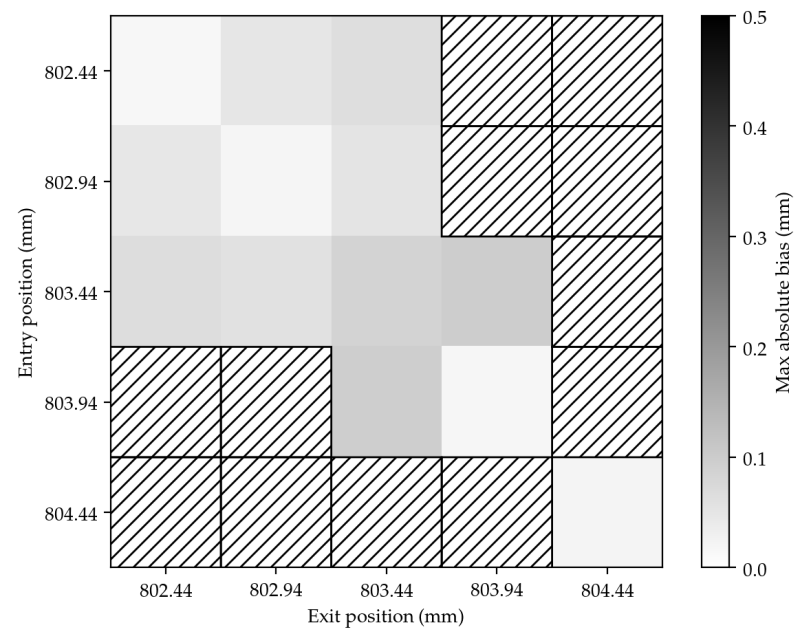

(b)

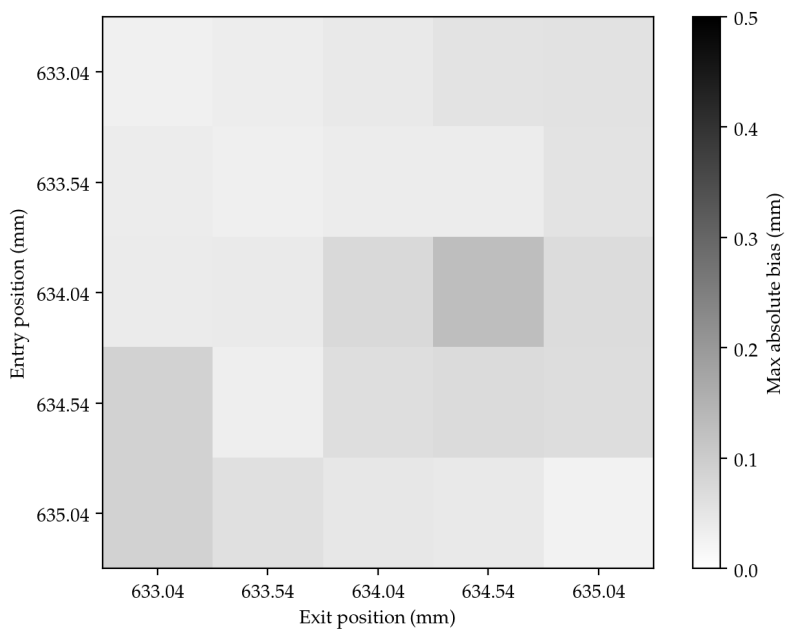

(c)

Figure 11: (a) Maximum bias in phantom (C) for different entry and exit positions, negative positions are in water, positive values are in bone. (b) and (c) Maximum bias reached near the air/head and the teeth/mandible interfaces, respectively. The hatched areas correspond to paths for which the number of protons was too small for statistical analysis. 
correct for the bias in a longitudinally heterogeneous phantom. Using ground-truth knowledge of the composition of phantom (B), we were able to correct the bias induced by the water assumption (Figure 7).

On another note, we have confirmed that the RMS is not a satisfactory metric to evaluate the impact of heterogeneities on the accuracy of the MLP. Indeed, the RMS error is impacted by the systematic bias in the MLP estimate as well as the uncertainty around this estimation. This latter uncertainty depends on the MCS in the medium (for example, there is more scattering in bone thus the RMS will be higher). Using other metrics such as the mean or maximum error (bias) allows for a better assessment of the MLP accuracy. Collins-Fekete et al. (2017) considered the fact that no difference was seen in terms of RMS between the formalism assuming water and their adapted formalism indicated that the increase of RMS in heteregeneous phantoms was only due to an increased scattering. Our results show this holds true for longitudinal heterogeneities : since there was no systematic error in the MLP (at least for straight line paths, and the bias for other trajectories was small compared with the one for transverse heterogeneities), the rise in the RMS was only caused by MCS, thus the use of the heterogeneous formalism did not reduce the RMS error. The fact that the RMS error did not decrease in phantom $(\mathrm{C})$ when using the heterogeneous formalism is because this formalism does not take into account transverse heterogeneities.

Our results also call into question the definition of the MLP in heterogeneous media. Usually, the MLP is defined as the junction of the most likely transverse positions determined independently as a function of depth. While this representation is a valid path in the case of a unimodal distribution of the proton positions, the set of most likely positions does not define a realistic and continuous path in the case of a distribution with more than one peak (Figure 10.). For such cases, MLP should better be understood as Most Likely Positions rather than Most Likely Path. From Figure 10k, we can imagine several competing paths. In this particular case, we know there are two main proton populations: the first one is composed of protons that stayed in the same medium - in this case, bone - and the second one of protons that transitioned in the water region. Therefore, the path associated with the first Gaussian (Eq. 3) corresponds to protons having spent their entire trajectory in the bone region, which explains its proximity to the theoretical path (the difference should be due to the water assumption only). The path from the second Gaussian is harder to characterize. It corresponds to protons that passed in the water region, however, as not all protons transition at the same depth, these protons do not all have the same trajectory. It might correspond to the most probable trajectory among all possible trajectories of protons passing to water.

It could be of interest to develop an alternative definition of the MLP without assuming a Gaussian distribution of the scattering angles and positions and taking into account heterogeneities. However, one must keep in mind that measured biases for realistic heterogeneities only reached $0.13 \mathrm{~mm}$ at the most. In addition, out of the many protons recorded and used for a full proton CT reconstruction, only a few will have entry and exit coordinates that suggest they have grazed a density interface. Therefore, a correction of the bias might not significantly improve the spatial resolution in $\mathrm{pCT}$.

We must also acknowledge that our results are entirely based on Monte Carlo simulations, and what we refer to as "real MLP" is only the result of simulated proton trajectories. This is because it would not have been possible to experimentally track proton trajectories inside a target. Therefore, these proton tracks depend on the physics models used in Geant 4 and on the simulation parameters. Regarding the step size, we have verified its choice does not affect the estimated real MLPs. A simulation such as the one used in Figure 4 was performed in phantom (C) using a maximum step size of $100 \mu \mathrm{m}$. The resulting maximum bias was of $0.27 \mathrm{~mm}$ while the one measured when using a step size of $5 \mathrm{~mm}$ was of $0.28 \mathrm{~mm}$. This indicates a step size of $5 \mathrm{~mm}$ is small enough to accurately measure the error on the MLP.

Besides, it might be interesting to investigate the effects of transverse heterogeneities on the 
protons' exit energy (see Figure 5 and 8) and whether they can lead to artifacts during the reconstruction as protons having the same theoretical MLP would end up with a wide range of different energy losses. We have shown that close to a transverse interface, the real MLP will lean towards the less dense material. In terms of tomographic reconstruction, this means that protons will be backprojected along a path in the dense material (e.g. bone) whereas they actually traveled partly in another tissue (water, air, etc.). Since these protons will have a higher energy given that they traversed a tissue with a smaller RSP (relative stopping power), the backprojected values in the region around the interface, and more particularly close to the dense tissue, will tend to be underestimated. We expect the reconstructed interface to be shifted, for example in Phantom (C), it will be higher than expected since energy values corresponding to protons traversing water will be backprojected in the bone region. In addition to this geometrical effect, the energy profiles suggest that some of the values used in reconstruction would not correspond to either material of the interface which will make the region around the interface blurry. We have yet to confirm theses assumptions, but the impact of transverse heterogeneities during reconstruction could explain some of the artifacts observed in other works. For example, Wang et al. (2010) noted a dark ring artifact around object boundaries on reconstructed images. While the authors explain this artifact by a problem with the Geant 4 energy loss model, it could be related to transverse heterogeneities.

Furthermore, energy profiles such as the ones shown in Figures 5 , 8 and 9 would complicate the energy cuts used to filter nuclear events. In (Schulte et al., 2008), $3 \sigma$ data cuts on the exit energy were proposed to eliminate inelastic nuclear collisions, but such cuts on the observed distributions would probably less efficient when the distribution is constituted of several peaks. In this case, it would be hard to distinguish between low energy protons that underwent nuclear collisions in water and protons that traversed mostly bone, for example.

\section{CONCLUSION}

We have demonstrated transverse heterogeneities induce non Gaussian spatial and angular distributions. When the distributions are merely skewed, this causes a biased prediction of the MLP as protons will be more likely to traverse the less dense medium. The maximum bias between the MLP calculated using the conventional formalism of Schulte et al. (2008) and the MLP derived from Monte Carlo tracks reaches $0.4 \mathrm{~mm}$ for a $20 \mathrm{~cm}$ water/bone interface, and $0.13 \mathrm{~mm}$ for a more realistic interface in a head phantom. When the distributions of the transverse positions are not unimodal, the notion of a single most likely path remain open to debate.

\section{Acknowledgments}

The work of Feriel Khellaf was partially supported by the project DIC20161236452 of the Fondation pour la Recherche Médicale (FRM). The work of Nils Krah was supported by funding from the European Union's Horizon 2020 research and innovation programme under the Marie Sklodowska-Curie grant agreement No 753370. This work was performed within the framework of the SIRIC LYriCAN INCa_INSERM_DGOS_12563 and the LABEX PRIMES (ANR-11-LABX0063) of Université de Lyon, within the program "Investissements d'Avenir" (ANR-11-IDEX0007) operated by the French National Research Agency (ANR).

\section{References}

Agostinelli, S. et al. (2003). "GEANT4 - a simulation toolkit". In: Nuclear instruments and methods in physics research section A: Accelerators, Spectrometers, Detectors and Associated Equipment 506.3, pp. 250-303. DOI: 10.1016/S0168-9002(03)01368-8. 
Arbor, $\mathrm{N}$ et al. (2015). "Monte Carlo comparison of x-ray and proton CT for range calculations of proton therapy beams". In: Physics in Medicine $\&$ Biology 60.19, p. 7585. DOI: 10.1088/ 0031-9155/60/19/7585

Bopp, C. et al. (2014). "The impact of tracking system properties on the most likely path estimation in proton CT". In: Physics in Medicine E Biology 59.23, N197. DOI: 10.1088/ 0031-9155/59/23/n197.

Collins-Fekete, C.-A. et al. (2017). "Extension of the Fermi-Eyges most-likely path in heterogeneous medium with prior knowledge information". In: Physics in Medicine $\mathcal{E}$ Biology 62.24, p. 9207. DOI: $10.1088 / 1361-6560 /$ aa955d.

Cormack, A. et al. (1976). "Quantitative proton tomography: preliminary experiments". In: Physics in Medicine 83 Biology 21.4, p. 560. DOI: 10.1088/0031-9155/21/4/007.

Eyges, L. (1948). "Multiple scattering with energy loss". In: Physical Review 74.10, p. 1534. DOI: $10.1103 /$ physrev.74.1534.

ICRP (2009). "Adult Reference Computational Phantoms. ICRP Publication 110". In: Annals of the ICRP. ICRP Publication 11039 (2). DOI: 10.1016/j.icrp.2009.09.001.

Jan, S et al. (2011). "GATE V6: a major enhancement of the GATE simulation platform enabling modelling of CT and radiotherapy". In: Physics in Medicine $\mathcal{E}$ Biology 56.4, p. 881. DoI: 10.1088/0031-9155/56/4/001.

Krah, N. et al. (2018). "Proton radiographies with a commercial range telescope detector using dedicated post processing methods". In: Physics in Medicine E Biology 63.20, p. 205016. DOI: $10.1088 / 1361-6560 / \mathrm{aae} 043$.

Li, T. et al. (2006). "Reconstruction for proton computed tomography by tracing proton trajectories: A Monte Carlo study". In: Medical physics 33.3, pp. 699-706. DOI: 10.1118/1.2171507.

Paganetti, H. (2012). "Range uncertainties in proton therapy and the role of Monte Carlo simulations". In: Physics in Medicine \& Biology 57.11, R99. DOI: 10.1088/0031-9155/57/11/r99.

Rit, S. et al. (2013). "Filtered backprojection proton CT reconstruction along most likely paths". In: Med Phys 40.3, 031103, p. 031103. DOI: 10.1118/1.4789589.

Schaffner, B et al. (1998). "The precision of proton range calculations in proton radiotherapy treatment planning: experimental verification of the relation between CT-HU and proton stopping power". In: Physics in Medicine \& Biology 43.6, p. 1579. DOI: 10.1088/00319155/43/6/016.

Schulte, R. et al. (2008). "A maximum likelihood proton path formalism for application in proton computed tomography". In: Medical physics 35.11, pp. 4849-4856. DOI: 10.1118/1.2986139.

Svensson, H. et al. (1984). "Report 35". In: Journal of the International Commission on Radiation Units and Measurements os18.2, pp. 152-154. DOI: 10.1093/jicru/os18.2.Report35. eprint: /oup/backfile/content_public/journal/jicru/os18/2/10.1093_jicru_os18. 2.152/2/jicruos18-0152.pdf.

Tanaka, S. et al. (2016). "Development of proton CT imaging system using plastic scintillator and CCD camera". In: Physics in Medicine \& Biology 61.11, p. 4156. DOI: 10.1118/1.4955578.

Tanaka, S. et al. (2018). "Improved proton CT imaging using a bismuth germanium oxide scintillator". In: Physics in Medicine \& Biology 63.3, p. 035030. DOI: 10.1088/1361-6560/ aaa515.

Wang, D. et al. (2010). "On the use of a proton path probability map for proton computed tomography reconstruction a". In: Medical physics 37.8, pp. 4138-4145. DOI: 10.1118/1. 3453767.

Wang, D. et al. (2011). "Bragg peak prediction from quantitative proton computed tomography using different path estimates". In: Physics in Medicine $\&$ Biology 56.3, p. 587. DOI: 10 . 1088/0031-9155/56/3/005.

West, D et al. (1972). "Radiography with $160 \mathrm{MeV}$ protons". In: Nature 239.5368, p. 157. DoI: $10.1038 / 239157 \mathrm{~b} 0$. 
West, D et al. (1973). "Proton-scattering radiography". In: Non-destructive Testing 6.5, pp. 249257. DOI: $10.1016 / 0029-1021$ (73) 90072-8.

Williams, D. (2004). "The most likely path of an energetic charged particle through a uniform medium". In: Physics in Medicine \&J Biology 49.13, p. 2899. DOI: 10.1088/0031-9155/49/ $13 / 010$.

Wong, K. et al. (2009). "The effect of tissue inhomogeneities on the accuracy of proton path reconstruction for proton computed tomography". In: AIP Conference Proceedings. Vol. 1099. AIP, pp. 476-480. DOI: 10.1063/1.3120078.

Yang, M. et al. (2012). "Comprehensive analysis of proton range uncertainties related to patient stopping-power-ratio estimation using the stoichiometric calibration". In: Physics in Medicine $\&$ Biology 57.13, p. 4095. DOI: 10.1088/0031-9155/57/13/4095.

Zhang, R. et al. (2017). "Improvement of single detector proton radiography by incorporating intensity of time-resolved dose rate functions". In: Physics in Medicine 8 Biology 63.1, p. 015030. DOI: 10.1088/1361-6560/aa9913. 